\title{
Automated Design of Microfluidics-Based Biochips: Connecting Biochemistry to Electronics CAD
}

\author{
Krishnendu Chakrabarty \\ Department of Electrical and Computer Engineering \\ Duke University \\ Durham, NC 27708, USA
}

\begin{abstract}
Microfluidics-based biochips offer exciting possibilities for highthroughput sequencing, parallel immunoassays, blood chemistry for clinical diagnostics, DNA sequencing, and environmental toxicity monitoring. The complexity of microfluidic devices is expected to become significant in the near future due to the need for multiple and concurrent biochemical assays on multifunctional and reconfigurable platforms. This paper presents early work on topdown system-level computer-aided design (CAD) tools for the synthesis, testing and reconfiguration of microfluidic biochips. Synthesis tools map behavioral descriptions to a droplet-based microfluidic biochip and generate an optimized schedule of assay operations, the binding of assay operations to functional units, and the layout and droplet flow-paths. Cost-effective testing techniques lead to the detection of manufacturing defects and operational faults. Reconfiguration techniques, incorporated in these CAD tools, can easily bypass faults once they are detected. Thus the biochip user can concentrate on the development of the nano- and micro-scale bioassays, leaving assay optimization and implementation details to design automation tools.
\end{abstract}

\section{Introduction}

Microfluidics-based biochips are revolutionizing laboratory procedures involving molecular biology $[1,2,3]$. Often refereed to as lab-on-a-chip or bio-MEMS, these devices automate highly repetitive laboratory tasks by replacing cumbersome equipment with miniaturized and integrated systems, and they enable the handling of small amounts, e.g., micro- and nano-liters, of fluids. Thus they are able to provide ultra-sensitive detection at significantly lower cost than traditional methods.

Advances in microfluidics technology offer exciting possibilities in the realm of enzymatic analysis (e.g., glucose and lactate assays), DNA analysis (e.g., PCR and nucleic acid sequence analysis), proteomic analysis involving proteins and peptides, immuno-assays, and environmental toxicity monitoring. An emerging application area for microfluidics-based biochips is clinical diagnostics, especially immediate point-of-care diagnosis of diseases $[4,5]$. Microfluidics-based devices, capable of continuous sampling and real-time testing of air/water samples for biochemical toxins and other dangerous pathogens, can serve as an always-on "bio-smoke alarm" for early warning $[6,7]$.

A popular category of microfluidic biochips containes permanently-etched micropumps, microvalves, and microchannels, and their operation is based on the principle of continuous fluid flow $[2,3]$ A promising alternative is to manipulate liquids as discrete droplets $[8,9]$. Following the analogy of microelectronics, this approach is referred to as "digital microfluidics". In contrast to continuous-flow biochips, digital microfluidics-based biochips offer scalable system architecture based on a two-dimensional microfluidic array of identical basic unit cells. Moreover, because each droplet can be controlled independently, these systems also have dynamic reconfigurability, whereby groups of unit cells in a microfluidic array can be reconfigured to change their functionality during the concurrent execution of a set of bioassays. The reconfigurability inherent in digital microfluidic biochips can therefore be utilized to achieve longer system lifetimes through online reconfiguration to avoid operational faults. It can also be used to increase production yield through production-time reconfiguration to bypass manufacturing defects.

As the use of microfluidics-based biochips increases, their complexity is expected to become significant due to the need for multiple and concurrent assays on the chip. Next-generation biochips are likely to be multifunctional and adaptive "biochemical processing" devices. For example, inexpensive biochips for clinical diagnostics offer high throughput with low sample volumes, and they integrate hematology, pathology, molecular diagnostics, cytology, microbiology, and serology onto the same platform. There is a need to deliver the same level of computer-aided design (CAD) support to the biochip designer that the semiconductor industry now takes for granted. CAD tools will reduce design cost, and they will allow designers to harness the new technology that is rapidly emerging for integrated biofluidics. They will obviate the need for tedious chip redesign by hand for each target application.

Efforts are underway in the CAD community to identify synergies between biochips and microelectronics CAD. The 2005 Design, Automation, and Test in Europe (DATE) Conference included a well-attended "Biochips Day" event. A full-day workshop was co-organized and co-located with DATE 2006. The IEEE Transactions on CAD published a special issue on biochips in February 2006. The IEEE Design \& Test magazine will publish a special issue on biochips in early 2007.

This paper presents early work on CAD tools for biochip design. The paper describes synthesis tools that map behavioral descriptions to a droplet-based microfluidic biochip and generate an optimized schedule of bioassay operations. These tools also determine the binding of assay operations to functional units, and the layout and droplet flow-paths for the biochip. Cost-effective testing techniques are presented to detect faults after manufacture and during field operation. It is shown how on-line and off-line reconfiguration techniques can be used to easily bypass faults once they are detected. Thus the biochip user can concentrate on the development of the nano- and micro-scale bioassays, leaving implementation details to design automation tools.

\section{Technology Issues}

Early biochips were based on the concept of a DNA microarray, which is a piece of glass, plastic or silicon substrate on which segments of DNA (probes) have been affixed in a microscopic array. There are a number of commercial microarrays available in the marketplace today, e.g., the GeneChip ${ }^{\circledR}$ DNAarray from Affymetrix, the DNA microarray from Infineon $\mathrm{AG}$, and the 
NanoChip ${ }^{\circledR}$ microarray from Nanogen [10-12]. Similar to a DNA microarray, a protein array is a miniature array where a multitude of different capture agents, most frequently monoclonal antibodies, are deposited on a chip surface (glass or silicon); they are used to determine the presence and/or amount of proteins in biological samples, e.g., blood. A drawback of DNA and protein arrays is that they are neither reconfigurable nor scalable after manufacture.

The basic idea of microfluidic biochips is to integrate all necessary functions for biochemical analysis onto one chip using microfluidics technology. These micro-total-analysis-systems ( $\mu$ TAS) are more versatile and complex than microarrays. Integrated functions include microfluidic assay operations and detection, as well as sample pre-treatment and preparation.

\subsection{Continuous-Flow Microfluidics}

These technologies are based on the manipulation of continuous liquid flow through microfabricated channels. Actuation of liquid flow is implemented either by external pressure sources, integrated mechanical micropumps, or by electrokinetic mechanisms [2, 3]. Continuous-flow systems are adequate for many well-defined and simple biochemical applications, and for certain tasks such as chemical separation, but they are less suitable for tasks requiring a high degree of flexibility or complicated fluid manipulations. These closed-channel systems are inherently difficult to integrate and scale because the parameters that govern flow field vary along the flow path making the fluid flow at any one location dependent on the properties of the entire system. Moreover, unavoidable shear flow and diffusion in microchannels makes it difficult to eliminate intersample contamination and dead volumes. Permanently-etched microstructures also lead to limited reconfigurability and poor fault tolerance capability.

\subsection{Droplet-Based Microfluidics}

Alternatives to the above closed-channel continuous-flow systems include novel open structures, where the liquid is divided into discrete, independently controllable droplets, and these droplets can be manipulated to move on a substrate. By using discrete unitvolume droplets, a microfluidic function can be reduced to a set of repeated basic operations, i.e., moving one unit of fluid over one unit of instance. This "digitization" method facilitates the use of a hierarchical and cell-based approach for microfluidic biochip design. Defect tolerance can be easily incorporated in the design due to the inherent dynamic reconfigurability. Therefore, in contrast to continuous fluid flow, digital microfluidics offers a flexible and scalable system architecture as well as high defect-tolerance capability.

A number of methods for manipulating microfluidic droplets have been proposed in the literature [13-15]. Of these, electrical methods to actuate droplets appear to be the most promising $[8,9$, 19]. Dielectrophoresis (DEP) and electrowetting-on-dielectric (EWOD) are the two most common electrical methods. DEP relies on the application of high-frequency AC voltages [22, 23], while EWOD is based on DC (or low-frequency AC) voltages [9, 10]. Excessive Joule heating is often seen as a problem for DEP actuation [19]. EWOD uses DC (or low-frequency AC) electric fields to directly control the interfacial energy between a solid and liquid phase. In contrast to DEP actuation, Joule heating is virtually eliminated in EWOD because the dielectric layer covering the electrodes blocks DC electric current.

EWOD microfluidics-based biochips: The basic unit cell of a EWOD-based "digital" microfluidic biochip consists of two parallel glass plates, as shown in Figure 1(a). The bottom plate contains a patterned array of individually controllable electrodes, and the top plate is coated with a continuous ground electrode. A dielectric insulator, e.g., parylene $\mathrm{C}$, coated with a hydrophobic film of Teflon $\mathrm{AF}$, is added to the plates to decrease the wettability of the surface and to add capacitance between the droplet and the control electrode. The droplet containing biochemical samples and the filler medium, such as the silicone oil, are sandwiched between the plates; the droplets travel inside the filler medium. In order to move a droplet, a control voltage is applied to an electrode adjacent to the droplet, and at the same time, the electrode just under the droplet is deactivated. By varying the electrical potential along a linear array of electrodes, electrowetting can be used to move nanoliter volume liquid droplets along this line of electrodes [21]. The velocity of the droplet can be controlled by adjusting the control voltage $(0 \sim 90 \mathrm{~V})$, and droplets can be moved at speeds of over $20 \mathrm{~cm} / \mathrm{s}$ [21]. Droplets can also be transported, in user-defined patterns and under clockedvoltage control, over a two-dimensional array of electrodes shown in Figure 2(b) without the need of micropumps and microvalves.

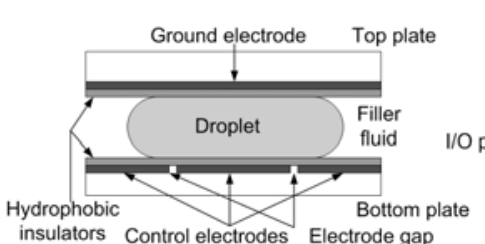

(a)

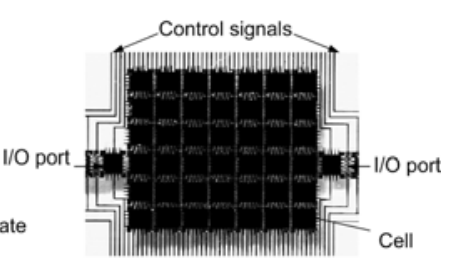

(b)
Figure 1: (a) Basic unit cell used in an EWOD-based digital microfluidic biochip; (b) a two-dimensional array for digital microfluidics.

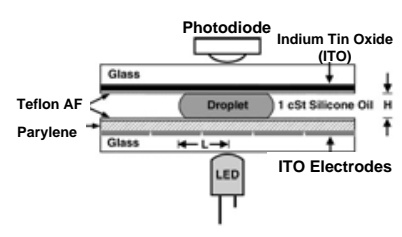

(a)

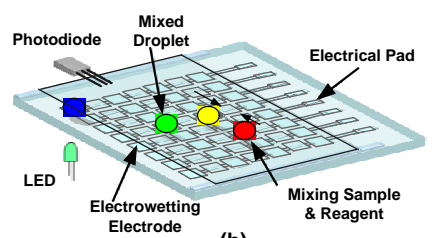

(b)
Figure 2: Schematic of a digital microfluidic biochip used for colorimetric assays: (a) basic unit cell; (b) Top view of microfluidic array.

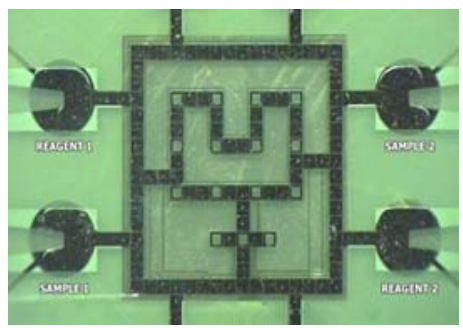

Figure 3: Fabricated microfluidic array used for multiplexed bioassays [6].

The in-vitro measurement of glucose and other metabolites, such as lactate, glutamate and pyruvate, is of great importance in clinical diagnosis of metabolic disorders. A colorimetric enzymekinetic glucose assay has been recently demonstrated on a digital microfluidic biochip [5]. This biochip integrates an optical detection system consisting of an LED and a photodiode; see Figure 2. In addition, glucose, lactate, glutamate and pyruvate assays can be combined to form a set of multiplexed bioassays that are performed concurrently on a microfluidic platform. Figure 3 illustrates a fabricated microfluidic system used for multiplexed bioassays [5]. 
There are natural similarities between digital microfluidic arrays and reconfigurable computing systems based on fieldprogrammable gate arrays (FPGAs). However, the "programmability" of FPGAs is limited by the well-defined roles of interconnect and logic blocks. Interconnect cannot be used for storing information and logic blocks cannot be used for routing. In contrast, the microfluidics architecture that we are developing offers significantly more programmability. The unit cells can not only be used for storage and functional operations, but also for transporting fluid droplets.

Reconfiguration techniques for microfluidic arrays are also fundamentally different from the redundancy-based methods (spare rows/columns) used for memories, processor arrays, and FPGAs. Due to the absence of programmable interconnects such as switches between microfluidic cells, a droplet is only able to move directly to the adjacent cells. This property of fluidic locality implies that the functionality of a faulty unit cell can only be assumed by its physically-neighboring cells in the array. Fluidic locality limits the reconfiguration capabilities of the spare rows/columns if they are not adjacent to the faulty cell.

\section{Design Automation Automation Trends and Challenges: From MEMS to Microfluidics}

MEMS design is a relatively young field compared to integrated circuit design. Since the concept of CAD systems for MEMS was first proposed at Transducer'87 [24], a number of commercial MEMS CAD tools have been developed [25, 26]. However, because of the differences in actuation methods between MEMS and microfluidics, they cannot be directly used for the design of microfluidic biochips. Some design automation techniques have been proposed for DNA probe arrays [27]; however, microfluidicsbased biochips are more versatile and complex than DNA arrays.

Current design methodologies for microfluidics-based biochips are typically full-custom and bottom-up in nature. Since much microfluidics work to date has been focused on device development, most design automation research for microfluidic biochips has been limited to device-level physical modeling of components [28-30]. Commercial computational fluid dynamics tools, such as CFD-ACE+ from CFD Research Corporation and FlumeCAD from Coventor, Inc. support 3D simulation of microfluidic transport. A droplet electrohydrodynamics framework to analyze DEP and EWOD is presented in [30].

Once the devices are optimized using detailed physical simulation, they can be used to assemble a complete microfluidicsbased biochip. Therefore, a bottom-up development approach is rather natural, which involves the development of each block from the device to the system level. Microfluidic devices (e.g., electrodes and glass plates) are combined to form microfluidic modules (e.g., mixers or storage units), which are then combined to obtain the complete system (e.g., microfluidics-based glucose detectors). Since the system behavior can only be verified at this late stage, costly and time-consuming redesign effort is required at the circuit level if the system does not satisfy design constraints.

Although these full-custom and bottom-up methodologies have been employed successfully in the past, they are clearly inadequate for the design of complex microfluidics-based biochips. While topdown system-level design tools are now commonplace in IC design, few such efforts have been reported for digital microfluidics-based biochips. A recent release of CoventorWare from Coventor, Inc. includes microfluidic behavioral models to allow top-down systemlevel design. However, this CAD tool is only able to deal with continuous flow systems, and it is therefore inadequate for the design of digital microfluidic biochips.

\section{Design Automation Methods for Digital Microfludics}

In this section, we provide an overview of the synthesis, test, and reconfiguration techniques that we have developed for digital microfluidics. Other related work is also discussed where appropriate.

\subsection{Synthesis and Reconfiguration Techniques}

We first decoupled architectural-level synthesis (i.e., high- level synthesis) from geometry-level synthesis (i.e., physical design) [31, 32]. Architectural-level synthesis for microfluidic biochips can be viewed as the problem of scheduling assay functions and binding them to a given number of resources so as to maximize parallelism, thereby decreasing response time. On the other hand, geometrylevel synthesis addresses the placement of resources and the routing of droplets to satisfy objectives such as area or throughput.

As in the case of high-level synthesis for integrated circuits, resource binding in the biochip synthesis flow refers to the mapping from bioassay operations to available functional resources. Note that there may be several types of resources for any given bioassay operation. For example, a $2 \times 2$-array mixer, a $2 \times 3$-array mixer and a $2 \times 4$-array mixer can be used for a droplet mixing operation, but with different mixing times. In such cases, a resource selection procedure must be used. On the other hand, resource binding may associate one functional resource with several assay operations; this necessitates resource sharing. Once resource binding is carried out, the time duration for each bioassay operation can be easily determined. Scheduling determines the start times and stop times of all assay operations, subject to the precedence and resource-sharing constraints.

A key problem in the geometry-level synthesis of biochips is the placement of microfluidic modules such as different types of mixers and storage units. Since digital microfluidics-based biochips enable dynamic reconfiguration of the microfluidic array during run-time, they allow the placement of different modules on the same location during different time intervals. A simulated annealing-based heuristic approach has been developed to solve the NP-complete problem in a computationally efficient manner [31]. Solutions for the placement problem can provide the designer with guidelines on the size of the array to be manufactured. If module placement is carried out for a fabricated array, area minimization frees up more unit cells for sample collection and preparation.

We have also developed a synthesis methodology that unifies operation scheduling, resource binding, and module placement [33]; see Figure 4. Exact placement information, instead of a crude area estimate, is used to judge the quality of architectural-level synthesis. This method allows architectural design and physical design decisions to be made simultaneously. In order to increase sampling efficiency, we also attempt to free up more array area for sample collection and preparation by minimizing the area of the chip devoted to analysis.

Efficient reconfiguration techniques have been developed to bypass faulty unit cells in the microfluidic array. A microfluidic module containing a faulty unit cell can easily be relocated to another part of the microfluidic array by changing the control voltages applied to the corresponding electrodes [32]. Defect tolerance can also be achieved by including redundant elements in the system; these elements can be used to replace faulty elements 


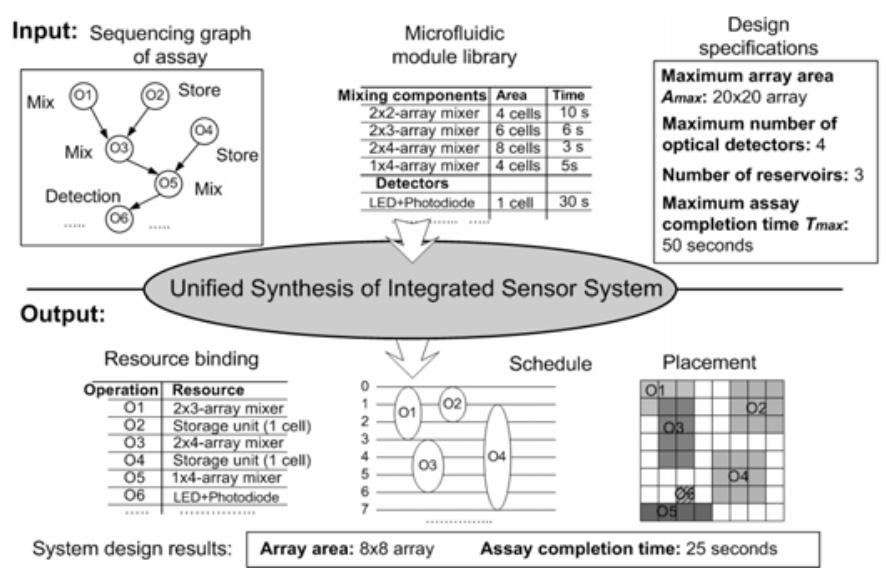

Figure 4: An example illustrating unified synthesis.

through reconfiguration techniques [34]. Another method is based on graceful degradation, in which all elements in the system are treated in a uniform manner, and no element is designated as a spare [35]. In the presence of defects, a subsystem with no faulty element is first determined from the faulty system. This subsystem provides the desired functionality, but with a gracefully-degraded level of performance (e.g., longer execution times). Due to the dynamic reconfigurability of digital microfluidics-based biochips, microfluidic components (e.g., mixers) can be viewed as reconfigurable virtual devices. For example, a $2 \times 4$ array mixer (implemented using a rectangular array of control electrodes - two in the X-direction and four in Y-direction) can easily be reconfigured to a $2 \times 3$ array mixer or a $2 \times 2$ array mixer.

Figure 5(a) shows the module placement results and the microfluidic array design for a representative protein assay [33], Figure 5(b) shows the corresponding results when some of the unit cells are array are faulty, and reconfiguration is used in an unified manner with synthesis. The solution obtained for the fault-free array yields a biochip design with a $9 \times 9$ microfluidic array and the completion time for the protein assay is 363 seconds. The design for the faulty array allows the protein assay to operate with an increase of only $6 \%$ in the completion time, i.e., the completion time is now 385 seconds.

\subsection{Testing Techniques and Design-for-Test (DFT)}

Fault modeling, fault simulation, and a DFT methodology for continuous-flow microfluidic systems have recently been proposed $[36,37]$. Limited work on the testing of "digital" microfluidic biochips has been reported to date.

We have classified faults in these systems as being either catastrophic or parametric [38]. Catastrophic (hard) faults lead to a complete malfunction of the system, while parametric (soft) faults cause a deviation in the system performance. A parametric fault is detectable only if this deviation exceeds the tolerance in system performance.

Catastrophic faults in digital biochips may be caused by a number physical defects, for example.:

- Dielectric breakdown: The breakdown of the dielectric at high voltage levels creates a short between the droplet and the electrode. When this happens, the droplet undergoes electrolysis, thereby preventing further transportation.

- Short between the adjacent electrodes: If a short occurs between two adjacent electrodes, the two electrodes effectively
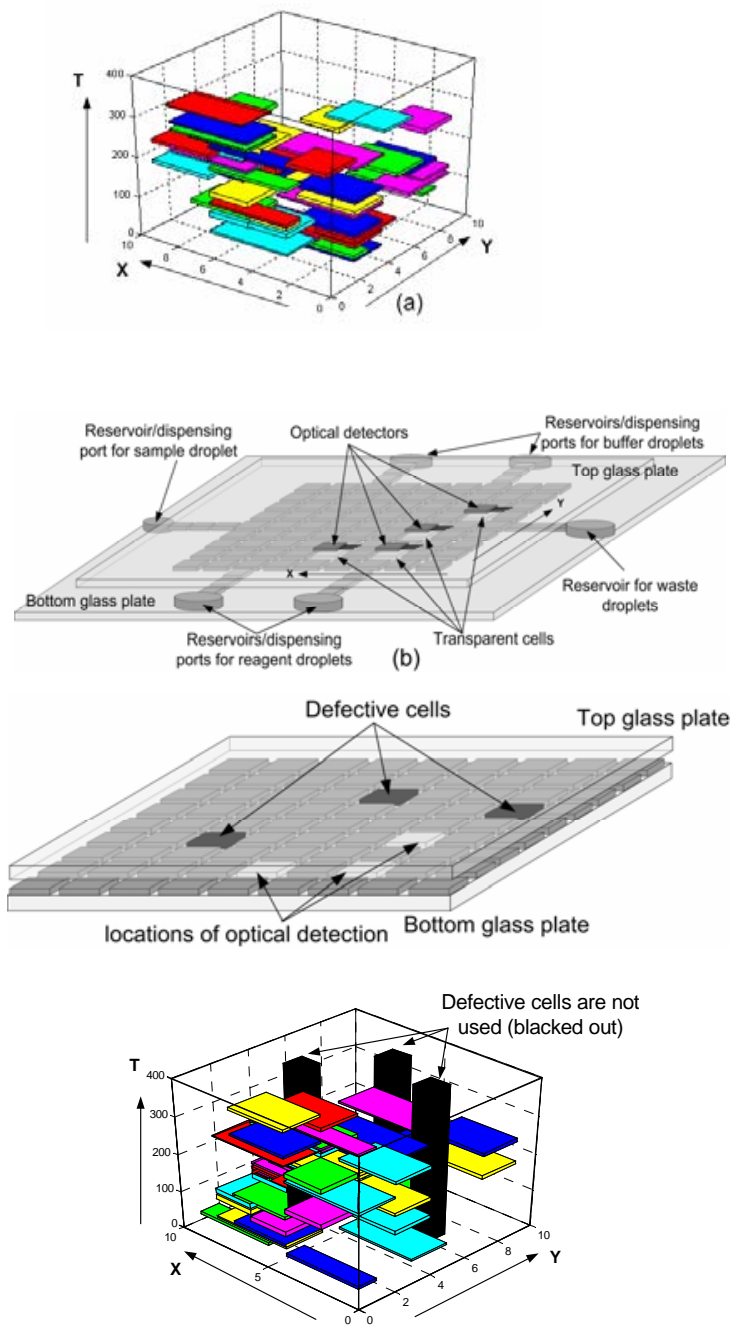

(c)

Figure 5. (a) A 3-D model illustrating the synthesis results; (b) a digital microfluidic biochip for a protein assay. (c) a defective array and module placement for the protein assay on this array.

form one longer electrode. When a droplet resides on this electrode, it is no longer large enough to overlap the gap between adjacent electrodes. As a result, the actuation of the droplet can no longer be achieved.

- Degradation of the insulator: This degradation effect is unpredictable and may become apparent gradually during the operation of the microfluidic system. A consequence is that droplets often fragment and their motion is prevented because of the unwanted variation of surface tension forces along their flow path.

- Open in the metal connection between the electrode and the control source: This defect results in a failure in activating the electrode for transport.

Physical defects that cause parametric faults include the following:

- Geometrical parameter deviation: The deviation in insulator thickness, electrode length and height between parallel plates may exceed their tolerance value. 
- $\quad$ Change in viscosity of droplet and filler medium. These can occur during operation due to an unexpected biochemical reaction, or changes in operational environment, e.g., temperature variation.

We have proposed a unified test methodology for digital microfluidic biochips, whereby faults can be detected by controlling and tracking droplet motion electrically [39, 40]. Test stimuli droplets containing a conductive fluid (e.g., KCL solution) are dispensed from the droplet source. These droplets are guided through the unit cells following the test plan towards the droplet sink, which is connected to an integrated capacitive detection circuit. Most catastrophic faults result in a complete cessation of droplet transportation [38]. Therefore, we can determine the faultfree or faulty status of the system by simply observing the arrival of test stimuli droplets at selected ports.

An efficient test plan ensures that testing does not conflict with the normal bioassay, and it guides test stimuli droplets to cover all the unit cells available for testing. We have formulated the test planning problem in terms of the graph partitioning and the Hamiltonian path problem [39]. With negligible hardware overhead, this method also offers an opportunity to implement self-test for microfluidic systems and therefore eliminate the need for costly, bulky, and expensive external test equipment. Furthermore, after detection, droplet flow paths for bioassays can be reconfigured dynamically such that faulty unit cells are bypassed without interrupting the normal operation.

\subsection{Droplet Routing Methods}

Droplet routing between modules, and between modules and $\mathrm{I} / \mathrm{O}$ ports (i.e., on-chip reservoirs), is a key problem in biochip physical design. We have recently developed a systematic routing method for digital microfluidic biochips [41]; the two-step approach in [41] attempts to minimize the number of cells used for droplet routing, while satisfying constraints imposed by performance goals and fluidic properties.

Wire routing is a well-studied problem in VLSI design. Due to the analogy between digital microfluidics and digital electronics, many classical VLSI routing techniques can be leveraged for the droplet routing problem. However, there exist some important differences. For example, whereas electrical nets must not be shortcircuited in VLSI routing, i.e., they cannot intersect each other, different droplet routes can be overlapped on some locations as long as they satisfy fluidic constraints. This is due to the property of virtual nets in digital microfluidic biochips, i.e., the droplet route is dynamically formed by sequentially activating the corresponding control electrodes. Consequentially, capacity constraints that result from fixed routing regions in VLSI design are not as important in droplet routing.

The problem of minimizing the length of droplet routes is analogous to the minimization of the total wire length in VLSI routing. The fluidic ports on the boundary of microfluidic modules are referred to as pins, and we assume that pin assignment has been done in the placement phase. Similarly, we refer to the droplet routes between pins of different modules or on-chip reservoirs as nets. Thus, a fluidic route on which a single droplet is transported between two terminals can easily be modeled as a 2-pin net. We also need to move two droplets from different terminals to one common microfluidic module (e.g., mixer) for mixing. To allow droplet mixing simultaneously during their transport, which is preferable for efficient assay operations, we need to model such fluidic routes using 3-pin nets, instead of two individual 2-pin nets. Moreover, unlike the classical VLSI routing problem, these 2-pin nets or 3-pin nets may overlap in some cells, thus also leading to the reduction of the total route length. To avoid unintended mixing between different droplets, however, multiple droplets that are routed should satisfy some important fluidic constraints.

Fluidic constraints: During droplet routing, a minimum spacing between droplets must be maintained to prevent accidental mixing, except for the case when droplet merging is desired (e.g., in 3-pin nets). For multiple droplet routes that may intersect or overlap with each other, fluidic constraint rules must be introduced to avoid undesirable behavior. Details are presented in [41].

Timing constraints: We need to ensure that the delay for each droplet route does not exceed some maximum value, e.g., $10 \%$ of a time-slot used in scheduling. Otherwise, the schedule obtained from the synthesis procedure is no longer valid. This timing constraint is analogous to the interconnect delay constraints in VLSI routing that require each wire net (or critical path) to meet its timing budget. Note that since a droplet may be held at a location in some time slots during its route, the delay for each droplet route is not identical to the route length. The delay for a droplet route therefore consists of the transport time as well as the idle time [41].

A channel routing problem for continuous-flow microfluidic biochips has been investigated in [42]. This routing problem is similar to the classical single-layer VLSI routing problem. In [43], the droplet path-planning problem for digital microfluidic biochips is modeled as a motion-planning problem with multiple moving robots. A drawback is that only routes between two terminals are considered. However, many droplet routes connect multiple terminals when practical bioassays are applied to the digital microfluidic platform. A second approach for coordinating droplets in digital microfluidic biochips has been presented in [43]. By viewing the microfluidic array as a network, the authors reduced the droplet path-planning problem to a network flow problem. Since droplet motion is only limited to the fixed streets, this approach does not exploit some of the important benefits of digital microfluidics, e.g., dynamic reconfigurability.

\subsection{Pin-Constrained Chip Design}

Design and CAD research for digital microfluidic biochips has mostly been focused on directly-addressable arrays. This method is adequate for small/medium-scale microfluidic electrode arrays (with fewer than $10 \times 10$ electrodes). However, the number of pins for a design based on direct addressing can be prohibitively high for a large array. For example, a total of 104 pins are needed to independently control the electrodes in a $100 \times 100$ array. Multilayer electrical connection structures and wire routing solutions are complicated by the large number of independent control pins in such arrays. Product cost, however, is a major marketability driver due to the one-time-use (disposable) nature of most emerging devices. Hence, simpler routing solutions are necessary so that the electrical wiring can be easily incorporated in a low-cost implementation.

Recently, multiplayer (PCB) technology has been proposed as a means to rapidly prototype and inexpensively mass-fabricate digital microfluidic biochips [45]. However, multiple metal layers for PCB designs may lead to reliability problems and they tend to increase fabrication cost. Thus, reduction of the number of independent control pins is important for its commercialization.

Pin-constrained design of digital microfluidic biochips was proposed and analyzed in [5]. The number of control pins for a fabricated electrowetting-based biochip is minimized by using a multi-phase bus for the fluidic pathways. Every $n^{\text {th }}$ electrode in an $n$-phase bus is electrically connected. Thus, only $n$ control pins are needed for a transport bus, irrespective of the number of electrodes that it contains. Although the multi-phase bus method is useful for 
reducing the number of control pins, it is only applicable to a onedimensional (linear) array.

An alternative method based on a cross-reference driving scheme is presented in [45]. This method allows control of an $N \times M$ grid array with only $N+M$ control pins. However, it requires a special electrode structure (i.e., both top and bottom plates containing electrode rows), which results in increased manufacturing cost for disposable microfluidic chips. Moreover, this design is not suitable for high-throughput assays because droplet movement is inherently slow.

More recently, a promising design method based on array partitioning has been proposed for pin-constrained biochips [46] The microfluidic array is divided into several partitions and sets of pins are determined, where each set of pins correspond to a partition and all the sets are of the same size. For example, if a biochip of arbitrary size is divided into six partitions and five pins are allocated per set, only $5 \times 6=30$ pins are needed to independently address the individual unit cells of the array. By carefully controlling the number of partitions, the total number of pins is reduced significantly compared to the direct-addressing scheme.

\section{Emerging Applications}

On-chip assays for determining the concentrations of target analytes is a natural application for digital microfluidics. The specific focus of work in this area has been on multiplexed assays, where multiple analytes can be measured in a single sample. Section 3 presented an overview of multiplexed biossays. The onchip process steps include the following: 1) pre-diluted sample and reagent loading into on-chip reservoirs; 2) droplet dispensing of analyte solutions and reagents; 3) droplet transport; 4) mixing of analyte solution and reagent droplets; and 5) reaction product detection. We next review other recent work in this area.

Kurita et al demonstrated a biosensor for real time monitoring of in vivo lactate and glucose sampled from a microdialysis probe from a rat's brain [47]. Urban et al presented a biosensor array on a printed circuit board to detect glucose and lactate for whole blood monitoring [48]. Gao et al recently described a biosensor array, integrated with plastic microfluidics, for measurement of glucose and oxygen in human blood. [49]. The first microfluidic device for enzyme assays by mixing nanoliter volumes of a substrate, enzyme, and an inhibitor using electrokinetic flow was reported in [50] Wang et al demonstrated enzymatic assays of glucose and ethanol simultaneously [51-52]. An integrated system based on centrifugal microfluidics and ion-selective optode membranes was demonstrated for the determination of potassium ions by Madou's group [53]. Seki et al have demonstrated colorimetric assays for glucose and albumin on a replica molded polydimethylsiloxane (PDMS) chip [54]. Ahn et al have demonstrated an ingenious way of pumping fluids with on-chip air reservoirs in a disposable plastic LoC for the detection of oxygen, glucose, and lactate from human blood [55].

On-chip detection of commercial-grade 2,4,6-trinitrotoluene (TNT) and pure 2,4-dinitrotoluene has been demonstrated [56]. Assays have been performed on an electrowetting chip in three steps: dispensing, electrowetting-enabled mixing, and colorimetric detection. This work holds promising applications for the detection of land mines, unexploded ordinance, and soil contaminants.

In the realm of environmental monitoring, automated on-chip measurement of airborne particulate matter has been proposed using a scanning droplet method [57, 58]. Target applications include quasi real time sampling of airborne contaminants and bioagents, and detection and quantification of nitroaromatic explosive particles.

On-chip sequencing by synthesis is another emerging application. The number of bases in Genbank has increased exponentially with a doubling period of approximately 18 months and currently contains about $3 \times 10^{10}$ bases, equivalent to the content of 10 human genomes. Achieving the productivity necessary for the continued exponential growth of sequence information will require new, scalable methods for sequencing. There are several competing technologies that have proposed the use of microfluidic technologies to reduce reagent costs, which, along with instrument cost, have emerged as the primary cost of Sanger-based sequencing. These technologies include, mass spectrometry [59], nanopore sequencing [60-62], sequencing-by-hybridization [63], sequencing-by-synthesis [64], and miniaturized electrophoretic methods [65]. Work is also underway at Duke University to evaluate the digital microfluidic platform to perform miniaturized sequencing by synthesis.

Finally, protein crystallization is another emerging application area for microfluidic biochips. The specific biological function of a protein is determined by the three-dimensional (3D) arrangement of the constituent amino acids. Therefore, their structure needs to be understood for effective protein engineering, bioseparations, rational drug design, controlled drug delivery, and for the design of novel enzyme substrates, activators, and inhibitors. A widely used method to study the 3D structure of proteins is to crystallize the proteins and determine the structure using X-ray diffraction. Protein crystallization is a multi-parametric process that involves the steps of nucleation and growth, where molecules are brought into a thermodynamically unstable and a supersaturated state. In order to "hit" upon the correct parameters for the crystallization of proteins, typically a very large number of experiments $\left(10^{3}-10^{4}\right)$ are required. Zheng et al. presented an elegant way of screening for protein crystallization using $4 \mathrm{~nL}$ droplets of protein [66]. The protein, buffer, and precipitant were driven simultaneously by three syringes into a single PDMS microfluidic channel into a flowing stream of oil. Hansen et al. have demonstrated the potential of microfluidics for protein crystallization by screening for 144 parallel conditions on the chip [67].

\section{Conclusions}

Microfluidics-based biochips are revolutionizing high-throughput sequencing, parallel immunoassays, blood chemistry for clinical diagnostics, DNA sequencing, and environmental toxicity monitoring, and many other applications. The complexity of these devices is expected to grow steadily as more promising applications are identified and mapped to the digital microfluidic platform. Nextgeneration lab-on-a-chip system are likely to be multifunctional and adaptive "biochemical processing" devices. This paper has presented an overview of early work on top-down system-level CAD tools for the synthesis, testing and reconfiguration of digital microfluidic biochips. Techniques for synthesis, module placement, testing and reconfiguration, droplet routing, and pin-constrained design have been described. These CAD tools will reduce design cost, and they will allow designers to effectively harness new technology that is rapidly emerging for integrated microfluidics. They will obviate the need for tedious chip redesign by hand for each target application.

\section{Acknowledgments}

A number of students and colleagues contributed to the work reported in this paper. The author thanks Fei Su, Tao Xu, William Hwang, Phil Paik, Dr. Vamsee Pamula, and Prof. Richard Fair. This 
work was supported by the National Science Foundation under grants IIS-0312352 and CCF-0541055.

\section{References}

[1] M. A. Burns et al., "An integrated nanoliter DNA analysis device", Science, vol. 282, pp. 484-487, 1998.

[2] T. Thorsen, S. Maerkl and S. Quake, "Microfluidic large-scale integration", Science, vol. 298, pp. 580-584, 2002

[3] E. Verpoorte and N. F. De Rooij, "Microfluidics meets MEMS", Proceedings of the IEEE, vol. 91, pp. 930-953, 2003.

[4] T. H. Schulte, R. L. Bardell and B. H. Weigl "Microfluidic technologies in clinical diagnostics", Clinica Chimica Acta, vol. 321, pp. 1-10, 2002.

[5] V. Srinivasan, V. K. Pamula, and R. B. Fair, "An integrated digital microfluidic lab-on-a-chip for clinical diagnostics on human physiological fluids," Lab on a Chip, pp. 310-315, 2004.

[6] H. F. Hull, R. Danila and K. Ehresmann, "Smallpox and bioterrorism: Public-health responses", Journal of Laboratory and Clinical Medicine, vol. 142, pp. 221-228, 2003.

[7] S. Venkatesh and Z. A. Memish, "Bioterrorism: a new challenge for public health", International Journal of Antimicrobial Agents, vol. 21, pp. 200-206, 2003.

[8] M. G. Pollack, R. B. Fair and A. D. Shenderov, "Electrowettingbased actuation of liquid droplets for microfluidic applications", Applied Physics Letters, vol. 77, pp. 1725-1726, 2000.

[9] S. K. Ch et al., "Toward digital microfluidic circuits: creating, transporting, cutting and merging liquid droplets by electrowettingbased actuation", Proc. IEEE MEMS Conf., pp. 32-52. 2002.

[10] Affymetrix GeneChip ${ }^{\circledR}$, http://www.affymetrix.com

[11] Infineon Electronic DNA Chip, http://www.infineon.com

[12] Nanogen NanoChip ${ }^{\circledR}$, http://www.nanogen.com

[13] S. Mutlu et al., "Enhanced electro-osmosis pumping with liquid bridge and field effect flow rectification", Proc. IEEE MEMS Conf., pp. 850-853, 2004.

[14] B. S. Gallardo et al., "Electrochemical principles for active control of liquids on submillimeter scales," Science, vol. 283, pp. 57-60, 1999.

[15] T. S. Sammarco and M. A. Burns, "Thermocapillary pumping of discrete droplets in microfabricated analysis devices," AI Che J., vol. 45, 350-366, 1999.

[16] G. N. Somero, "Proteins and temperature", Annual Review of Physiology, vol. 57, pp.43-68, 1995.

[17] A. Wixforth and J. Scriba, "Nanopumps for programmable biochips", http://www.advalytix.de

[18] M. Washizu, "Electrostatic actuation of liquid droplets for microreactor applications," IEEE Trans. Ind. Appl., vol. 34, pp. 732-737, 1998.

[19] T. B. Jones et al., "Dielectrophoretic liquid actuation and nanodroplet formation," J. Appl. Phys., vol. 89, pp. 1441-1448, 2001.

[20] J. Vykoukal et al., "A programmable dielectric fluid processor for droplet-based chemistry", Micro Total Analysis Systems 2001, 7274, 2001.

[21] M. G. Pollack, A. D. Shenderov and R. B. Fair, "Electrowettingbased actuation of droplets for integrated microfluidics", Lab on a Chip, vol. 2, pp. 96-101, 2002.

[22] V. Srinivasan, V. K. Pamula, M. G. Pollack, R. B. Fair, "A digital microfluidic biosensor for multianalyte detection", Proc. IEEE MEMS Conf., pp. 327-330, 2003.

[23] V. Srinivasan, V. K. Pamula, M. G. Pollack, and R. B. Fair, "Clinical diagnostics on human whole blood, plasma, serum, urine, saliva, sweat, and tears on a digital microfluidic platform", Proc. $\mu$ TAS, pp. 1287-1290, 2003.

[24] S. Senturia, "Microfabricated structures for the measurement of mechanical properties and adhesion of thin films", Proc. Int. Conf. Sold-State Sensors and Actuators (Transducers), pp. 11-16, 1987.

[25] G. K. Fedder and Q. Jing, "A hierarchical circuit-level design methodology for microelectromechinal system", IEEE Trans. Circuits and Systems II, vol. 46, pp.1309-1315, 1999.
[26] S. K. De and N. R Aluru, "Physical and reduced-order dynamic analysis of MEMS", Proc. IEEE/ACM Int. Conf. Computer Aided Design, pp. 270-273, 2003.

[27] A. B. Kahng et al., "Evaluation of placement techniques for DNA probe array layout", Proc. IEEE/ACM Int. Conf. Computer Aided Design, pp. 262-269, 2003.

[28] A. N. Chatterjee and N.R. Aluru, "Combined circuit/device modeling and simulation of integrated microfluidic systems", Journal of Microelectromechanical Systems, vol. 14, pp. 81-95, 2005.

[29] B. Shapiro et al., "Modeling of electrowetted surface tension for addressable microfluidic systems: dominant physical effects, material dependences, and limiting phenomena" Proc. IEEE Conf. MEMS, pp. 201- 205, 2003.

[30] J. Zeng and F. T. Korsmeyer, "Principles of droplet electrohydrodynamics for lab-on-a-chip", Lab on a Chip, vol. 4, pp. 265-277, 2004

[31] F. Su and K. Chakrabarty, "Architectural-level synthesis of digital microfluidics-based biochips", Proc. IEEE International Conference on CAD, pp. 223-228, 2004.

[32] F. Su and K. Chakrabarty, "Module placement for fault-tolerant microfluidics-based biochips", ACM Transactions on Design Automation of Electronic Systems, vol. 11, pp. 682-710, 2006.

[33] F. Su and K. Chakrabarty, "Unified high-level synthesis and module placement for defect-tolerant microfluidic biochips", Proc. IEEE/ACM Design Automation Conference, pp. 825-830, 2005.

[34] F. Su and K. Chakrabarty, "Defect tolerance for gracefullydegradable microfluidics-based biochips", Proc. IEEE VLSI Test Symposium, pp. 321-326, 2005.

[35] F. Su and K. Chakrabarty, "Yield enhancement of reconfigurable microfluidics-based biochips using interstitial redundancy", $A C M$ Journal on Emerging Technologies in Computing Systems, vol. 2, April 2006 (to appear).

[36] H. G. Kerkhoff and H. P. A. Hendriks, "Fault modeling and fault simulation in mixed micro-fluidic microelectronic Systems", Journal of Electronic Testing: Theory and Applications, vol. 17, pp. 427-437, 2001.

[37] H. G. Kerkhoff and M. Acar, "Testable design and testing of microelectro-fluidic arrays", Proc. IEEE VLSI Test Symposium, pp. 403409, 2003.

[38] F. Su, S. Ozev and K. Chakrabarty, "Ensuring the operational health of droplet-based microelectrofluidic biosensor systems", IEEE Sensors Journal, vol. 5, pp. 763-773, August 2005.

[39] F. Su, S. Ozev and K. Chakrabarty, "Test planning and test resource optimization for droplet-based microfluidic systems", Journal of Electronic Testing: Theory and Applications, vol. 22, pp. 199-210, April 2006.

[40] F. Su, S. Ozev and K. Chakrabarty, "Concurrent testing of digital microfluidics-based biochips", ACM Transactions on Design Automation of Electronic Systems, vol 11, pp. 442-464, April 2006.

[41] F. Su, W. Hwang and K. Chakrabarty, "Droplet routing in the synthesis of digital microfluidic biochips", Proc. DATE Conf., pp. 323-328, 2006.

[42] A. J. Pfeiffer, T. Mukherjee, S. Hauan, "Synthesis of Multiplexed Biofluidic Microchips", IEEE Trans. CAD, February 2006.

[43] K.F. Böhringer, "Modeling and Controlling Parallel Tasks in Droplet-based Microfluidic Systems." IEEE Trans CAD, vol. 25, pp. 329-339, Februray 2006

[44] E. J. Griffith et al., "Performance characterization of a reconfigurable planar array digital microfluidic system", IEEE Trans on CAD, vol. 25, pp. 340-352, February 2006.

[45] J. Gong, and C.J. Kim, "Two-dimensional digital microfluidic system by multi-layer printed circuit board", Proc. IEEE MEMS Conf., 2005.

[46] W. Hwang, F. Su and K. Chakrabarty, "Automated design of pinconstrained digital microfluidic arrays for lab-on-a-chip applications", Proc. DAC, 2006.

[47] R. Kurita et al., "Microfluidic device integrated with pre-reactor and dual enzyme-modified microelectrodes for monitoring in vivo glucose and lactate," Sensors and Actuators B, 87, 296-303, 2002. 
[48] G. Urban, G. Jobst, and I. Moser, "Chemo- and biosensor microsystems for clinical applications," Proc. of SPIE (Chemical Microsensors and Applications), 3539, 46-50, 1998.

[49] C. Gao, J. Choi, M. Dutta, S. Chilukuru, J. Nevin, J. Lee, M Bissell, and C. Ahn, "A fully integrated biosensor array for measurement of metabolic parameters in human blood," Proc. $2^{\text {nd }}$ International IEEE-EMBS Special Topic Conference on Microtechnologies in Medicine \& Biology, 223-226, 2002.

[50] A.G. Hadd, D.E. Raymond, J.W. Halliwell, S.C. Jacobson, and J.M. Ramsey, "Microchip device for performing enzyme assays," Analytical Chemistry, 69, 3407-3412, 1997.

[51] J. Wang, M.P. Chatrathi, and B. Tian, "Microseparatin chips for performing multienzymatic dehydrogenase/oxidase assays: simultaneous electrochemical measurement of ethanol and glucose," Analytical Chemistry, 73, 1296-1300, 2001.

[52] J. Wang, M.P. Chatrathi, B. Tian, and R. Polsky, "Microfabricated electrophoresis chips for simultaneous bioassays of glucose, uric acid, ascorbic acid, and acetaminophen," Analytical Chemsitry, 72, 2514-2518, 2000

[53] R. Johnson et al., "Development of a Fully Integrated Analysis System for Ions Based on Ion-Selective Optodes and Centrifugal Microfluidics," Analytical Chemistry, 73, 3940-3946, 2001.

[54] M. Seki, R. Aoyama, J. Hong, T. Fujii, and I. Endo, "Multiple diagnostic analyses by enzymatic and chemical reaction on a PDMS microchip," Proc. $1^{\text {st }}$ International IEEE-EMBS Special Topic Conference on Microtechnologies in Medicine \& Biology, 21-24, 2000.

[55] C.H. Ahn et al., "Disposable smart lab on a chip for point of care diagnostics," Proceedings of the IEEE, 92, no. 1, 154-173, 2004.

[56] K. Pamula et al., "A droplet-based lab-on-a-chip for colorimetric detection of nitroaromatic explosives", Proc. IEEE MEMS Conf., 2005.

[57] R.B. Fair et al., "Integrated Chemical/Biochemical Sample Collection, Pre-concentration, and Analysis on a Digital Microfluidic Lab-on-a-Chip Platform," in Lab-on-a-Chip: Platforms, Devices, and Applications, L.A. Smith and D. Sobek, eds., Proc. SPIE, vol. 5591, pp. 113-124, Dec. 8, 2004.

[58] Y. Zhao and S.K. Cho, "Microparticle sampling by electrowettingactuated droplet sweeping," Lab on a Chip, vol. 6, 137-144, 2006.

[59] C. Jurinke, D. van den Boom, et al., "The use of MassARRAY technology for high throughput genotyping," Adv Biochem Eng Biotechnol., vol. 77, pp. 57-74, 2002.

[60] D.W. Deamer and M. Akeson, "Nanopores and nucleic acids: prospects for ultrarapid sequencing," Trends Biotechnol. vol. 18, pp. $147-151,2000$

[61] D.W. Deamer and D. Branton, "Characterization of nucleic acids by nanopore analysis," Acc Chem Res., vol. 35, pp. 817-825, 2002.

[62] J. Li, M. Gershow, et al., "DNA molecules and configurations in a solid-state nanopore microscope," Nat Mater vol. 2, pp. 611-615, 2003.

[63] S. Brenner, S.R. Williams, et al., "In vitro cloning of complex mixtures of DNA on microbeads: physical separation of differentially expressed cDNAs," Proc. Natl. Acad. Sci. USA. 97(4), pp. 1665-70, 2000.

[64] .L. Metzker, R. Raghavachari, et al., "Termination of DNA synthesis by novel 3'-modified-deoxyribonucleoside 5'triphosphates," Nucleic Acids Research 22(20), pp. 4259-67, 1994.

[65] C.A. Emrich, "Microfabricated 384-lane capillary array electrophoresis bioanalyzer for ultrahigh-throughput genetic analysis", Anal. Chem, 74, pp. 5076-5083, 2002.

[66] B. Zheng, L.S. Roach, R.F. Ismagilov, "Screening of protein crystallization conditions on a microfluidic chip using nanoliter-size droplets," Journal of American Chemical Society, 125, 11170$11171,2003$.

[67] C.L. Hansen et al., "A robust and scalable microfluidic metering method that allows protein crystal growth by free interface diffusion," Proceedings of the National Academy of Sciences, 99, 16531-16536, 2002. 